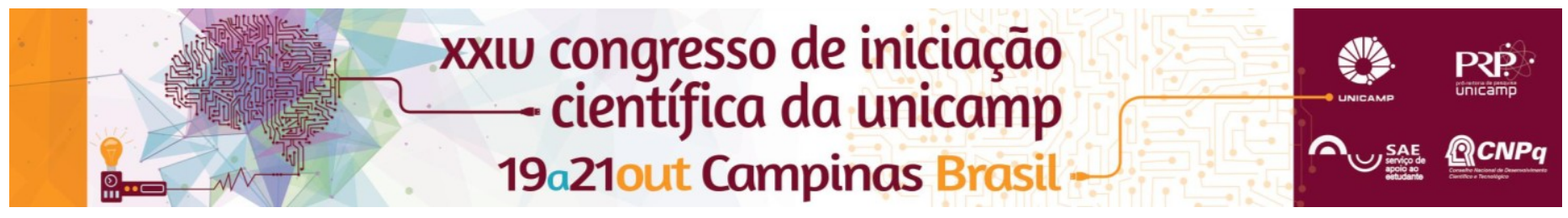

\title{
Avaliação de letramento em saúde em pacientes diabéticos tipo dois.
}

\author{
Paula da C. Pereira*, Stella. H. Hilkner, Beatriz A. Seignemartin, Elizabeth João Pavin e Maria C. R. Parisi.
}

\begin{abstract}
Resumo
O Diabetes Mellitus tipo 2 (DM2) manifesta-se quando o organismo passa a não ser capaz de usar adequadamente a insulina que produz, ou não produz insulina suficiente para o controle da taxa glicêmica; para que os pacientes tenham um autocuidado efetivo é necessário que eles sejam capazes de processar informações, com isso tomar decisões baseadas naquilo que foi compreendido; portanto este estudo teve como objetivo avaliar o letramento em 40 pacientes diabéticos tipo 2, seguidos em hospital terciário, utilizando o instrumento Short Assessment Of Health Literacy For Portuguese-Speaking Adults (Sahlpa-18),os resultados evidenciaram letramento em saúde inadequado.
\end{abstract}

\section{Palavras-chave: Autocuidado, Diabetes mellitus tipo 2, Letramento em saúde,}

\section{Introdução}

O Diabetes Mellitus tipo 2 (DM2) manifesta-se quando o organismo passa a não ser capaz de usar adequadamente a insulina que produz, ou não a faz suficiente para o controle da taxa glicêmica ${ }^{1}$. Sendo a forma de diabetes mais comum, representa $90-95 \%$ dos diabéticos ${ }^{1,2}$. O controle e tratamento da doença varia de acordo com a gravidade, podendo ser controlado com atividade física e planejamento alimentar em alguns casos e em outros necessitando do uso de insulina e/ou outros medicamentos ${ }^{1}$. O letramento diz respeito à habilidade que indivíduos têm de apreender e processar informações, com isso tomar decisões ${ }^{3}$. Quando este é aplicado à saúde ganha o nome de letramento funcional em saúde e refere-se ao grau de compreensão e capacidade de tomada de ação a partir de instruções e informações básicas para a manutenção da saúde ${ }^{4}$. Em 2010, uma Unidade Básica Distrital de Saúde de um município no interior de São Paulo, realizou estudo com 123 pacientes com DM2 e deste número, $87 \%$ eram alfabetizados, mas a maioria obteve resultados insatisfatórios em relação à compreensão e adesão ao auto cuidado ${ }^{5}$. Estudos nos EUA e Europa sugeriram que pacientes com letramento em saúde de baixo nível tinham maiores gastos com o custeamento da saúde, além de fazerem uso de variados serviços de maneira ineficiente, e precisarem de um maior suporte médico ${ }^{6}$. Este trabalho tem como objetivo avaliar o letramento em 40 pacientes diabéticos tipo 2 .

\section{Resultados e Discussão}

No DM2 há uma necessidade de grande participação dos pacientes no manejo da doença e quando há letramento em saúde insuficiente não detectado, estes pacientes enfrentam dificuldades no controle da doença ${ }^{7,8}$, pois sujeitos que possuem pouca capacidade de leitura muitas vezes se sentem acanhados e encobrem sua falta de compreensão, ocasionando num problema de entendimento das orientações, prescrições ou rótulos de medicamentos ${ }^{9}$.

Para a avaliação de letramento em pacientes diabéticos tipo 2 acompanhados em hospital terciário, utilizamos 0 instrumento SAHLPA-18, que avaliou pronúncia e compreensão de termos médicos comuns para estimar o nível de alfabetismo em saúde de adultos $^{10}$. Estudamos 40 pacientes diabéticos entre 50 e 75 anos, de ambos os sexos, com diagnóstico clínico e DOI: 10.19146/pibic-2016-51841 laboratorial de DM2 e seguimento no Ambulatório de Endocrinologia do Hospital de Clínicas - UNICAMP $\geq 1$ ano. A média das pontuações obtidas pelos 40 pacientes foi de 13,3; sendo que a pontuação máxima é 18, pontuações entre 0 e 14 sugerem que 0 letramento em saúde é inadequado ${ }^{10}$.

\section{Conclusões}

Os resultados obtidos mostraram letramento inadequado, este achado leva à reflexão dos impactos deste aspecto no autocuidado destes pacientes. Ao mesmo tempo, nos incentiva a pensar formas de ampliar e facilitar, não somente a comunicação como o empoderamento dos pacientes para que estes possam ampliar seu autocuidado de forma efetiva.

1. Diretrizes da sociedade Brasileira de Diabetes 2014-2015.

2. Gross JL., Silveiro SP., Camargo JL., Reichelt AJ., Azevedo MJ. de. Diabetes Melito: Diagnóstico, Classificação e Avaliação do Controle Glicêmico. Arq Bras Endocrinol Metab [Internet]. 2002 Feb [cited 2016 June 29] ; 46( 1 ): 16-26. Available from: http://www.scielo.br/scielo.php?script=sci_arttext\&pid=S0004-2730200200 $0100004 \& \operatorname{lng}=$ en

3. MICHAELIS: moderno dicionário da língua portuguesa. São Paulo: Companhia Melhoramentos, 1998 - (Dicionários Michaelis).

4. Passamai MPB. Letramento funcional em saúde: reflexões e conceitos sobre seu impacto na interação entre usuários, profissionais e sistema de saúde. [Internet]. 2012 Jun [cited 2016 June 29]. Available from: http://www.scielo.br/pdf/icse/2012nahead/aop2812.

5. Rodrigues FFL, Santos MA, Teixeira CRS, Gonela JT, Zanetti ML. Relação entre conhecimento, atitude, escolaridade e tempo de doença em indivíduos com diabetes mellitus. Acta paul. enferm. [Internet]. 2012 [cited 2016 July 12] ; 25( 2 ): 284-290. Available from: http://www.scielo.br/scie lo.php?script=sci_arttext\&pid=S0103-21002012000200020\&lng=en.

6. Franzen J, Mantwill S, Rapold R, Schulz PJ. The relationship between functional health literacy and the use of the health system by diabetics in Switzerland. Eur J Public Health. 2013 Dec 23. DOI: 10.1093/eurpub/ckt202 7. Chen GD, Huang CN, Yang YS, Lew-Ting CY. Patient perception of understanding health education and instructions has moderating effect on glycemic control. BMC Public Health. 2014 Jul 4;14:683.

8. Carvalho RBN, Deus ZLC, Silva JG, Silva ARV, Carvalho GCN. Educação em saúde na adesão ao tratamento por pacientes diabéticos. Rev EnfermUFPI. 2013 Jul-Sep; 2(3):33-9.

9. Parker R. Health literacy: a challenge for American patients and their health care providers. Health Promotion International. 2000; 15(4):277-83.

10. Apolinario D, Braga RCOP, Magaldi RM, Busse AL, Campora F, Brucki S et al . Short Assessment of Health Literacy for Portuguese-speaking Adults. Rev. Saúde Pública [Internet]. 2012 Aug [cited 2015 Nov 16] ; 46( 4 ): 7027.11 Available from: http://www.scielo.br/scielo.php ?script=sci_arttext\&pid=S0034-89102012000400015\&lng=en. Epub July 10, 2012. http://dx.doi.org/10.1590/S0034-89102012005000047. 